\title{
Is There a Higher-Order Mode Coupling Transition in Polymer Blends?
}

\author{
Angel J. Moreno, 1 a and Juan Colmenero ${ }^{1,2,3}$ \\ ${ }^{1}$ Donostia International Physics Center, Paseo Manuel de Lardizabal 4, 20018 San Sebastián, Spain. \\ ${ }^{2}$ Dpto. de Física de Materiales, Universidad del País Vasco (UPV/EHU), Apdo. 1072, 20080 San Sebastián, Spain. \\ ${ }^{3}$ Unidad de Física de Materiales, Centro Mixto CSIC-UPV, Apdo. 1072, 20080 San Sebastián, Spain.
}

(Dated: June 26, 2018)

\begin{abstract}
We present simulations on a binary blend of bead-spring polymer chains. The introduction of monomer size disparity yields very different relaxation times for each component of the blend. Competition between two different arrest mechanisms, namely bulk-like dynamics and confinement, leads to an anomalous relaxation scenario for the fast component, characterized by sublinear time dependence for mean squared displacements, or logarithmic decay and convex-to-concave crossover for density-density correlators. These anomalous dynamic features, which are observed over time intervals extending up to four decades, strongly resemble predictions of Mode Coupling Theory for nearby higher-order transitions. Chain connectivity extends anomalous relaxation over a wide range of blend compositions.
\end{abstract}

PACS numbers: 64.70.Pf, 83.80.Tc, 83.10.Rs

\section{INTRODUCTION}

The widespread potential applications of polymer blends have spurred a great deal of recent research on the dynamics of these systems showing tunable properties. Dynamics in polymer blends are directly related with one fundamental question of polymerbased soft matter systems: the way local friction arises in complex environments that are chemically heterogeneous. Thermodynamically miscible polymer blends exhibit complex dynamics. They show 'dynamic heterogeneity' in the meaning that two different segmental dynamics ( $\alpha$-relaxation) can be observed in the blend. Moreover, a symmetric broadening of any relaxation function of each component is usually observed as the glass transition of the blend is approached. These features are usually understood in terms of self-concentration effects induced by chain connectivity [1] and thermally driven concentration fluctuations [2].

For most of the investigated systems, the two components in the blend display qualitatively similar dynamic features. However, recent experimental results by nuclear magnetic resonance [3] and neutron scattering [4] suggest that a rather different picture emerges when the two blend components exhibit very different segmental mobilities in the homopolymer state and the concentration of the fast component in the blend is rather dilute. Then, the segmental dynamics of the fast component strongly deviate from the expected behavior and seem to be qualitatively different from that of the slow component. Moreover, fully atomistic molecular dynamics simulations (MDS) suggest the possibility of an unusual logarithmic time decay for the intermediate scattering function of the fast component [4].

Simulations are a valuable tool for investigating the microscopic origin of these unusual features. With present computational resources, a systematic study -i.e, by exploring a wide range of control parameters as temperature or blend composition - can only be performed by using very simple models, providing they display the essential dynamic features observed in real systems. With these ideas in mind, in this article we present an extensive MDS investigation on a blend of bead-spring polymer chains with components of very different mobilities. The fast component exhibits an anomalous relaxation scenario rather different from that characteristic of usual liquid-glass transitions. Namely, mean squared displacements display sublinear behavior, and density-density correlators exhibit a convex-to-concave crossover, which is observed both by varying wavevectors and control parameters. By properly tuning the latters, a logarithmic decay can be obtained.

These anomalous features are observed at intermediate time intervals extending up to four decades, and display striking analogies with Mode Coupling Theory (MCT) predictions for states close to a higher-order $\mathrm{MCT}$ transition, which emerges as the result from the competition between different arrest mechanisms we suggest bulk-like caging and confinement for the present case.

The article is organized as follows. In Section II we introduce the model and give computational details. In Section III we present simulation results and evidence that the fast component displays unusual relaxation features. In Section IV the framework of the Mode Coupling Theory is used in an operational way to describe simulation results. Conclusions are given in Section V.

\section{MODEL AND SIMULATION DETAILS}

We have carried out simulations on a model very similar to one originally introduced by Grest and Kremer 5 for homopolymers. Here we investigate a binary mixture of bead-spring polymer chains. Each chain consists of $N=10$ identical monomers of the species A or B. Monomers of both species have equal mass $m=1$. The interaction between two given monomers, placed on different chains or in a same one, is given by a soft-sphere potential plus a quadratic term: 
where $\epsilon=1$ and $\alpha, \beta \in\{\mathrm{A}, \mathrm{B}\}$. The interaction is zero beyond a cutoff distance $c \sigma_{\alpha \beta}$, with $c=1.15$. The values $C_{0}=7 c^{-12}$ and $C_{2}=6 c^{-14}$ guarantee continuity of potential and forces at the cutoff distance. The radii of the soft potential for the different types of interaction are $\sigma_{\mathrm{BB}}=1, \sigma_{\mathrm{AA}}=\delta \sigma_{\mathrm{BB}}$, and $\sigma_{\mathrm{AB}}=\left(\sigma_{\mathrm{AA}}+\sigma_{\mathrm{BB}}\right) / 2$. The potential (11) is purely repulsive. It does not show local minima within the interaction range $r<c \sigma_{\alpha \beta}$. Hence, slow dynamics in the present model arises as a consequence of steric effects, as in the computationally more involved case of chains of hard-sphere monomers.

Adjacent monomers of a same chain also interact through a finite extensible nonlinear elastic potential (FENE) [5, 6]:

$$
V_{\alpha \alpha}^{\mathrm{FENE}}(r)=-k R_{0}^{2} \epsilon \ln \left[1-\left(R_{0} \sigma_{\alpha \alpha}\right)^{-2} r^{2}\right],
$$

where $k=15$ and $R_{0}=1.5$. The superposition of the potentials (11) and (2) provides an effective bond potential for adjacent monomers with a sharp minimum at $r=0.985 \sigma_{\alpha \alpha}$, which makes bond crossing impossible for the investigated values of the monomer size disparity $\delta$.

The blend composition is defined as $x_{\mathrm{B}}=$ $N_{\mathrm{B}} /\left(N_{\mathrm{A}}+N_{\mathrm{B}}\right)$, with $N_{\mathrm{A}}$ and $N_{\mathrm{B}}$ denoting respectively the number of A- and B-chains. Data here presented for different compositions correspond to a single value $\delta=1.6$. Simulations were also carried out for values $1<\delta<1.75$ at a single composition $x_{\mathrm{B}}=0.5$. For $\delta \lesssim 1.2$ we did not observe signatures of the anomalous relaxation features exposed below.

The packing fraction, $\phi$, is defined as $\phi=$ $(\pi / 6) L^{-3}\left[N_{A} \sigma_{A A}^{3}+N_{B} \sigma_{B B}^{3}\right]$, with $L$ the side of the simulation box. In the following, temperature, $T$, distance and time, $t$, will be given respectively in units of $\epsilon / k_{B}, \sigma_{B B}$ and $\sigma_{B B}(m / \epsilon)^{1 / 2}$. Simulations have been carried out at a constant packing fraction $\phi=0.53$. This value is comparable to those used in simulations of slow relaxation in simple liquids [7].

We investigate the $T$-dependence of the dynamics for blend compositions in the range $0.1 \leq x_{B} \leq 1$. The total number of chains at each composition varies between 250 and 600 , with a minimum of 60 for the minoritary component. The system is prepared by placing the chains randomly in the simulation box, with a constraint that avoids core overlapping. Periodic boundary conditions are implemented. Equations of motion are integrated by using the velocity Verlet scheme [8], with a time step ranging from $2 \times 10^{-4}$ to $5 \times 10^{-3}$, for respectively the highest and the lowest temperature. A link-cell method [8] is used for saving computational time in the determination of the particles within the interaction range of a given one.

At each state point, the system is thermalized at the requested temperature by periodic velocity rescaling. After reaching equilibrium, energy, pressure, chain radii of gyration and end-to-end distances show no drift. Likewise, dynamic correlators and mean squared displacements show no aging, i.e., no time shift when being evaluated for progressively longer

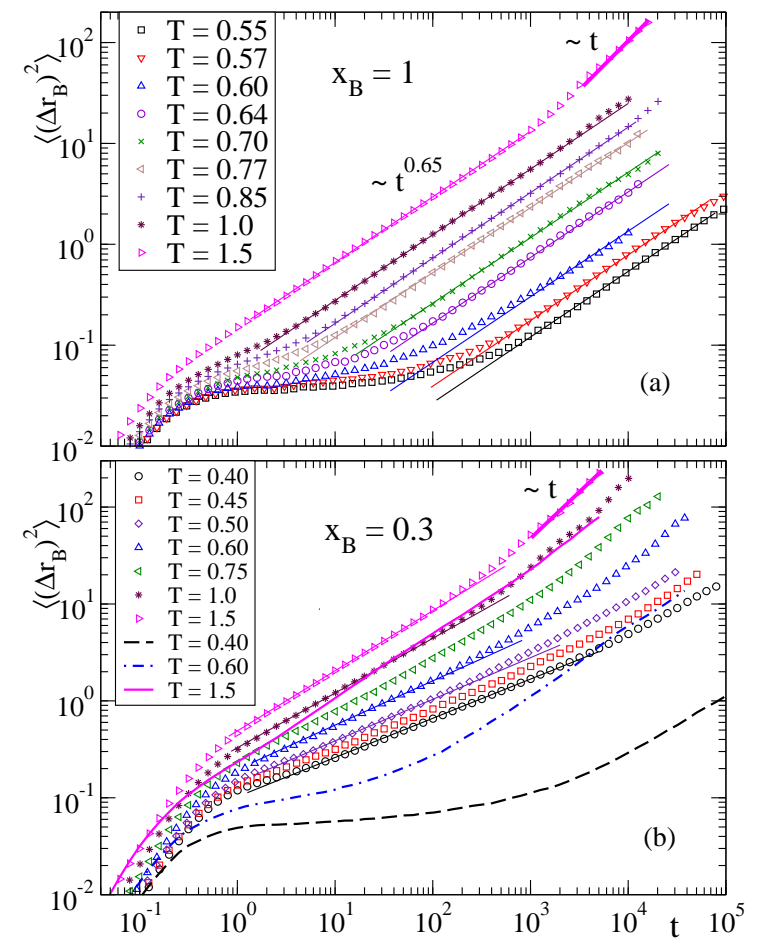

FIG. 1: Symbols: mean squared displacement of Bparticles for the homopolymer (a) and for a blend composition $x_{\mathrm{B}}=0.3(\mathrm{~b})$. Thick straight lines correspond to the linear diffusive regime $\left\langle\left(\Delta r_{\mathrm{B}}\right)^{2}\right\rangle \propto t$. Thin straight lines in panel (a) correspond to sublinear behavior $\left\langle\left(\Delta r_{\mathrm{B}}\right)^{2}\right\rangle \propto$ $t^{0.65}$. Thin straight lines in panel (b) correspond to $\left\langle\left(\Delta r_{\mathrm{B}}\right)^{2}\right\rangle \propto t^{\alpha}$ with a $T$-dependent exponent $\alpha$. From top to down, $\alpha=0.635,0.58,0.47,0.44$ and 0.415 . Results for A-particles (curves) at $x_{\mathrm{B}}=0.3$ are also shown for comparison. The exponent for the observed intermediate sublinear regime is 0.66 .

time origins. Once the system is equilibrated, a microcanonical run is performed for production of configurations, from which dynamic correlators and mean squared displacements are computed. For each state point, the latter quantities are averaged over typically 20-40 independent samples.

\section{SIMULATION RESULTS}

\section{a) Mean squared displacements}

Fig. 17 shows the $T$-dependence of the mean squared displacement of the B-particles, $\left\langle\left(\Delta r_{\mathrm{B}}\right)^{2}\right\rangle$, for the homopolymer case $\left(x_{\mathrm{B}}=1\right)$. We observe features analogous to similar bead-spring models previously investigated [6]. A plateau is developed after the initial ballistic regime $(t \lesssim 0.2)$, extending over longer times with decreasing temperature. This plateau corresponds to the well-known caging regime -i.e., the temporary trapping of each particle induced by their neighbors - observed when approaching a liquid-glass transition 9, 10. At longer times a crossover to a Rouse-like [6, 11] sublinear regime $\left\langle\left(\Delta r_{\mathrm{B}}\right)^{2}\right\rangle \propto t^{0.65}$ occurs, before the final crossover to linear diffusion at very long times, which is reached only for the highest investigated temperatures. The $T$-independent expo- 
nent 0.65 is compatible with previous simulations of similar models of bead-spring homopolymers [6].

Fig. 10 shows results for A- and B-particles for a blend composition $x_{\mathrm{B}}=0.3$. Monomer size disparity introduces a clear separation in the time scale of both components. Hence, on average, the big A-particles and the small B-ones are respectively slow and fast particles. A-particles exhibit features analogous to those observed in the homopolymer case. It is noteworthy that this observation is fulfilled in the whole composition range, suggesting that bulk-like dynamics is the only relevant arrest mechanism for the slow component. However, in the range $x_{\mathrm{B}} \lesssim 0.8$, B-particles exhibit a rather different behavior, as illustrated in Fig. 1b for a composition $x_{\mathrm{B}}=0.3$. The arrest mechanism is characterized by a larger localization length as compared to the homopolymer or to A-particles. This is evidenced by the larger value of the mean squared displacement at the inflection point after the end of the ballistic regime. A direct crossover from the ballistic regime to sublinear behavior $\left\langle\left(\Delta r_{\mathrm{B}}\right)^{2}\right\rangle \propto t^{\alpha}$ occurs, but differently from the homopolymer or the Aparticles, the exponent $\alpha$ is not constant, but shows a clear decrease with decreasing $T$. At sufficiently high temperature, again $\alpha \sim 0.6,-$ i.e., above a certain $T$ there are no essential differences between the transport mechanism for both species. However, for the lowest investigated temperatures we find $\alpha \sim 0.4$.

\section{b) Density-density correlators}

We compute partial density-density correlators $F_{\alpha \beta}(q, t)=\left\langle\rho_{\alpha}(q, t) \rho_{\beta}(-q, 0)\right\rangle /\left\langle\rho_{\alpha}(q, 0) \rho_{\beta}(-q, 0)\right\rangle$, with $\rho_{\alpha}(q, t)=\Sigma_{j} \exp \left[i \mathbf{q} \cdot \mathbf{r}_{\alpha, j}(t)\right]$, the sum extending over all the particles of the species $\alpha \in\{\mathrm{A}, \mathrm{B}\}$. Remarkable differences between the homopolymer and the blend are observed for density-density correlators for B-B pairs, $F_{\mathrm{BB}}(q, t)$. Figs. 2a and 2] show simulation results as a function of $T$ respectively for the homopolymer and for a blend composition $x_{\mathrm{B}}=0.3$. The wavevector $q$ corresponds to the maximum of the total static structure factor. Figs. 3 and $3 \mathrm{~b}$ show the corresponding $q$-dependence at two selected low values of $T$. The homopolymer exhibits the standard behavior in the proximity of liquid-glass transitions 6, 9, 10, 11, 12. After the initial transient regime, $F_{\mathrm{BB}}(q, t)$ shows a first decay to a plateau. With decreasing temperature, the plateau extends over longer time intervals. At long times, a second decay occurs from the plateau to zero. This second decay corresponds to the $\alpha$-process of the glass transition and is well described by a stretched exponential function. Analogous results are obtained for $F_{\mathrm{AA}}(q, t)$ in the whole composition range, as expected from the results above presented for the mean squared displacement of A-particles.

Rather different features are observed for $F_{\mathrm{BB}}(q, t)$ for compositions $x_{\mathrm{B}} \lesssim 0.8$. As can be seen in Fig. 3b, $F_{\mathrm{BB}}(q, t)$ does not exhibit a defined plateau at any value of $q$ or $T$. Moreover, an unusual convexto-concave crossover, both in $q$ and $T$, is observed for the shape of the correlators, and in particular

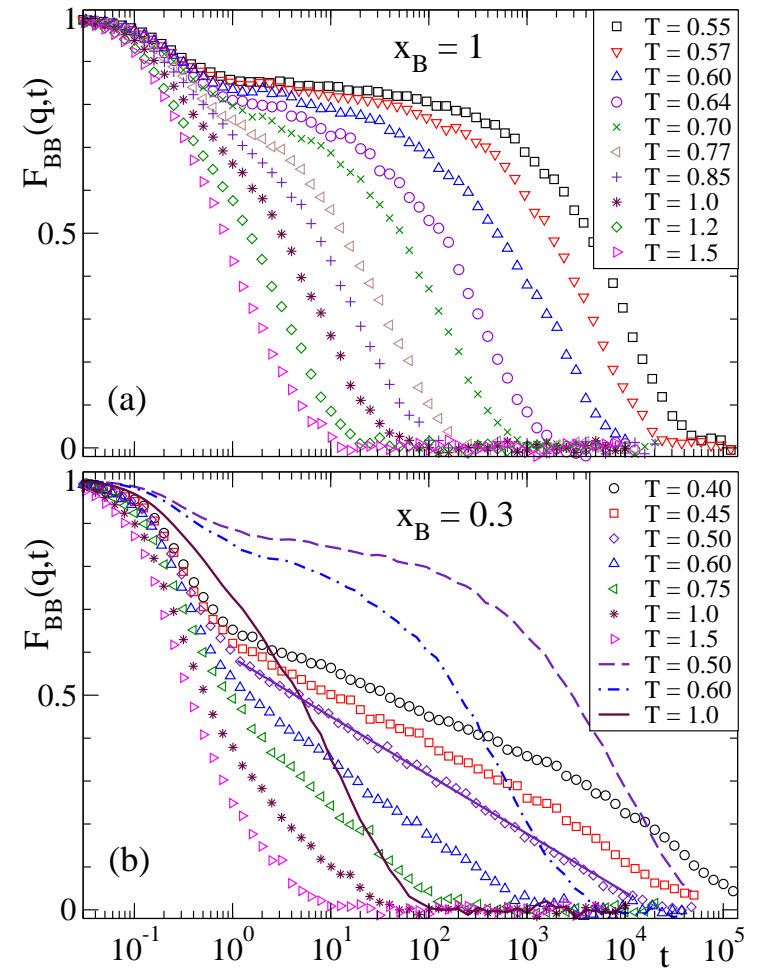

FIG. 2: Symbols in panels (a) and (b): T-dependence of $F_{\mathrm{BB}}(q, t)$, respectively for the homopolymer and for a blend composition $x_{\mathrm{B}}=0.3$, as computed from the simulations. The straight line in panel (b) indicates logarithmic decay over four time decades. For $x_{\mathrm{B}}=0.3$, the correlator $F_{\mathrm{AA}}(q, t)$ is also shown (curves) for comparison. The wavevectors are $q=7.1$ for the homopolymer and $q=4.5$ for $x_{\mathrm{B}}=0.3$, both values corresponding to the maxima of the respective total static structure factors.

there are state point-dependent values of $q$ for which logarithmic relaxation occurs over time intervals extending up to four decades. Recent atomistic simulations [4], at a single temperature and composition, of (fast) poly(ethylene oxide), PEO, in a (slow) matrix of poly(methyl methacrylate) report an impressively similar crossover at the $q$-dependence for selfcorrelations of PEO hydrogens (see Fig. 8 in Ref. 4), supporting the simple bead-spring model for polymer blends here investigated.

\section{DISCUSSION}

The unusual features observed for mean squared displacements and dynamic correlators of the fast component strongly resemble those reported by simulations 13, 14, 15] and experiments 16, 17, 18] for hard-sphere colloids with short-ranged attractive interactions. For these systems, such features have been recently rationalized in terms of the MCT 15, 19, 20, 21, 22, 23]. Motivated by this fact, we discuss the present results by using MCT in an operational way. In its ideal version -i.e., not including activated hopping events - MCT predicts 9, 10, 12] a sharp transition from an ergodic liquid to a nonergodic arrested state ('glass') at a given value $x_{\mathrm{c}}$ of the relevant control parameter $x$ (in practice $\phi$ or $T$ ). 


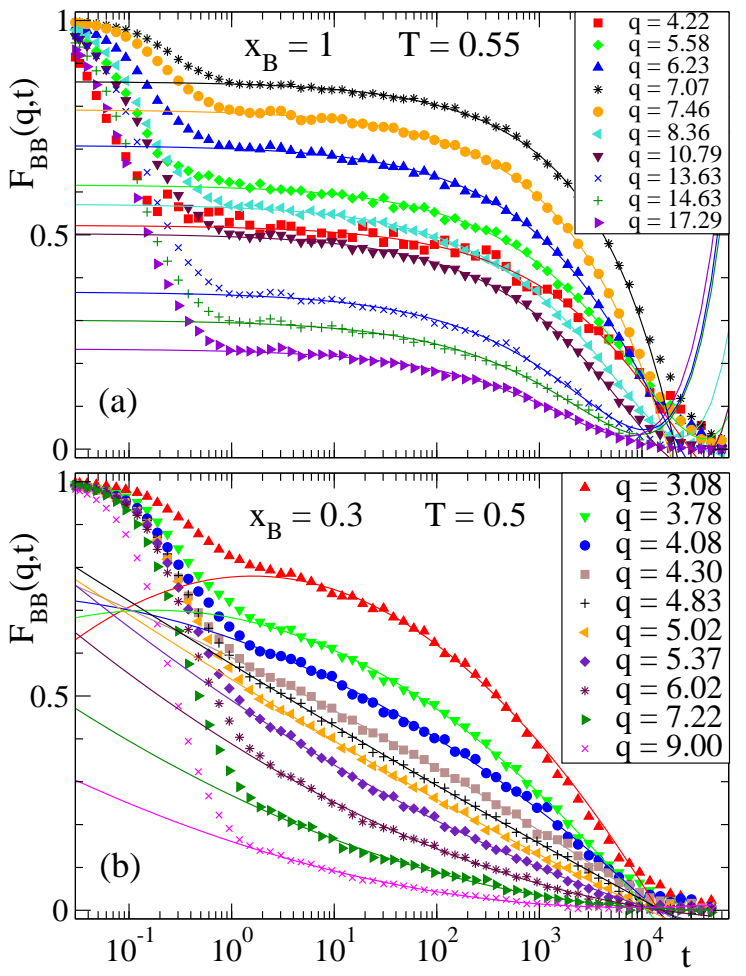

FIG. 3: Symbols in panels (a) and (b): $q$-dependence of $F_{\mathrm{BB}}(q, t)$, respectively for the homopolymer and for a blend composition $x_{\mathrm{B}}=0.3$, as computed from the simulations at two selected low temperatures. Curves in panels (a) and (b) are respectively fits to Eqs. (3) and (4).

When crossing the transition point from the ergodic to the arrested state, the long-time limit of the densitydensity correlator for wavevector $q, F(q, t)$, jumps discontinuously from zero to a finite value, denoted as the critical non-ergodicity parameter, $f_{q}^{c}$. In the MCT formalism, the standard liquid-glass transition is of the fold type (also denoted as $\mathrm{A}_{2}$ ) [9, 10, 12, 24]. The initial part of the $\alpha$-process (von Schweidler regime) is approximated by a power law expansion [9, 10, 12]:

$$
F(q, t) \approx f_{q}^{c}-h_{q}(t / \tau)^{b}+h_{q}^{(2)}(t / \tau)^{2 b},
$$

where the prefactors $h_{q}$ and $h_{q}^{(2)}$ only depend on the wavevector $q$. The characteristic time $\tau$ only depends on the separation parameter $\left|x-x_{\mathrm{c}}\right|$ and diverges at the transition point. The exponent $b$ is related to the so-called exponent parameter $1 / 2 \leq \lambda<1$, via the relation $\lambda=\Gamma^{2}(1+b) / \Gamma(1+2 b)$, with $\Gamma$ the Gamma function $[9,10,12$.

As previously found for similar bead-spring models 6, 11], Eq. (31) provides a good description for the decay from the plateau in the homopolymer case (see Fig. B ). A value $b=0.477$ is obtained. The corresponding exponent parameter is $\lambda=0.799$. The value of the constant exponent $\alpha=0.65$ for the observed Rouse-like regime in the mean squared displacement is consistent with MCT theoretical calculations for chains of $N=10$ hard-sphere monomers $(\alpha=0.6)$ 25. The obtained $q$-modulation for $f_{q}^{\mathrm{c}}$ is also consistent with MCT expectations $[26]$ and previous inves-

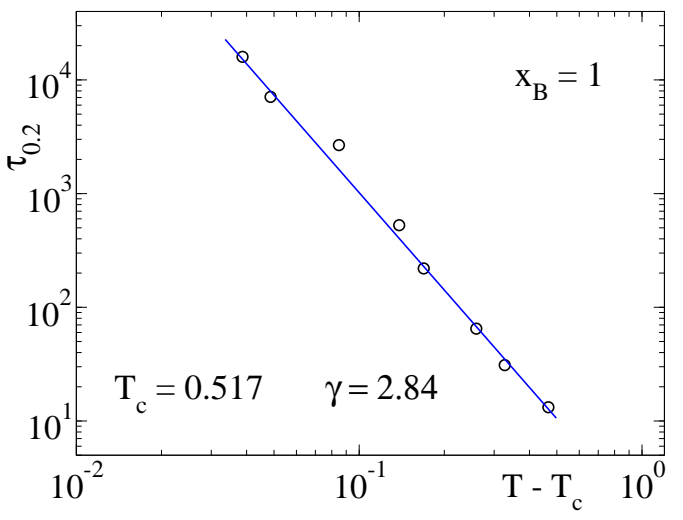

FIG. 4: For the homopolymer $\left(x_{\mathrm{B}}=1\right)$, temperature dependence of the $\alpha$-relaxation time of $F_{\mathrm{BB}}(q, t)$ at $q$ corresponding to the maximum of the static structure factor. Symbols are simulation data. The line is a fit to a power law $\tau_{0.2} \propto\left(T-T_{\mathrm{c}}\right)^{-\gamma}$ (see text).

tigations of bead-spring models 11.

Fig. 4 shows, for the homopolymer, the $T$ dependence of the relaxation time of $F_{\mathrm{BB}}(q, t)$, for $q$ at the maximum of the static structure factor. We define the former as the time $\tau_{0.2}$ where $F_{\mathrm{BB}}(q, t)$ decays to 0.2 , a value well below the plateau. This corresponds to times probing the $\alpha$-relaxation. According to MCT for $\mathrm{A}_{2}$-transitions [9, 10, 12], such times diverge at the transition point as a power law $\propto\left|x-x_{\mathrm{c}}\right|^{-\gamma}$ (in the present case $x=T$ ). The exponent $\gamma$ is related to $\lambda$ through the expressions $\gamma=1 / 2 a+1 / 2 b$ and $\lambda=\Gamma^{2}(1-a) / \Gamma(1-2 a)$. From the value $\lambda=0.799$ given above we obtain $\gamma=2.84$. Consistently, a good description of the data is obtained by forcing this value of $\gamma$ and leaving $T_{\mathrm{c}}$ as a free parameter (see Fig. 4). We obtain $T_{\mathrm{c}}=0.517$.

The features observed for B-particles in the composition range $x_{\mathrm{B}} \lesssim 0.8$ cannot be rationalized within the picture of a MCT $\mathrm{A}_{2}$-transition. However, they exhibit a striking resemblance with $\mathrm{MCT}$ predictions in the proximity of higher-order transitions $\mathrm{A}_{n+1}$, characterized by $\lambda=1$, which can emerge as the result from the interplay between $n \geq 2$ control parameters $x_{1}, x_{2} \ldots x_{n}$. Higher-order MCT transitions were initially derived for schematic models 27, 28], and later for short-ranged attractive colloids 19, 20, 21, 22, 23. as a first realization in real systems. Close to a higherorder transition, or more generally to a fold transition with $\lambda \lesssim 1$, an anomalous relaxation scenario emerges. The mean squared displacement exhibits an intermediate sublinear regime [22, 29] with a decreasing exponent as the transition is approached. As reported above, this behavior is observed for $B$-particles for blend compositions $x_{\mathrm{B}} \lesssim 0.8$. In the higherorder MCT scenario, $F(q, t)$ does not exhibit a defined plateau. Instead, in an intermediate time interval of several decades, it is approximated by a logarithmic expansion 22, 27, 28, 30]:

$$
F(q, t) \approx f_{q}^{\mathrm{c}}-H_{q} \ln (t / \tau)+H_{q}^{(2)} \ln ^{2}(t / \tau),
$$

where the prefactors $H_{q}$ and $H_{q}^{(2)}$ depend on $q$ and 


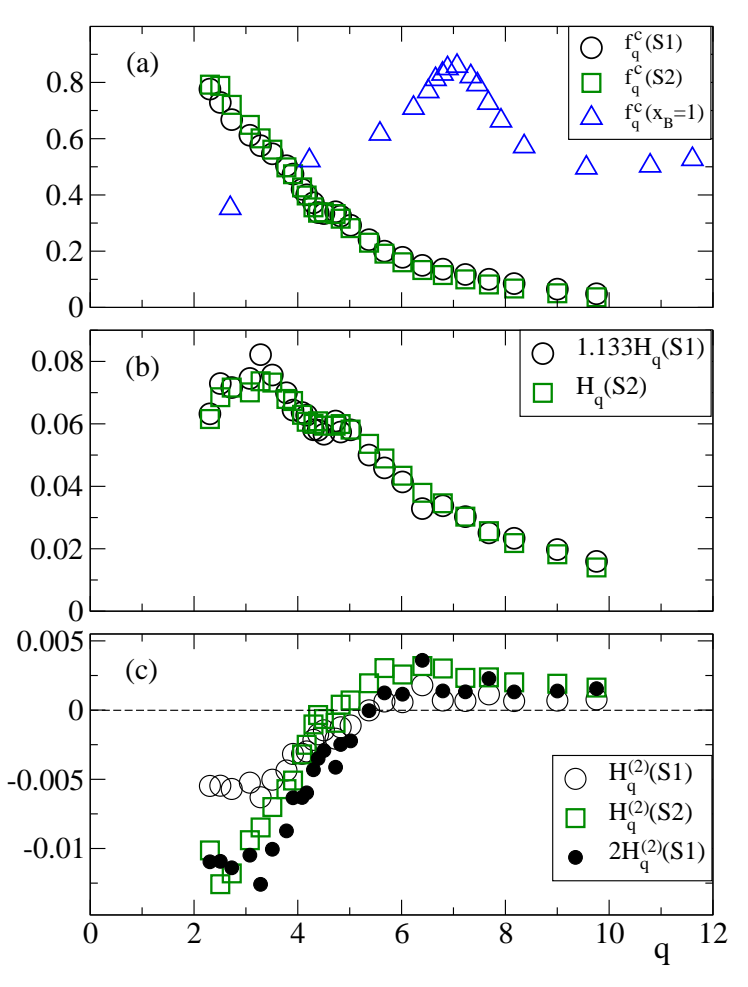

FIG. 5: Panels (a), (b), and (c) are respectively the values of $f_{q}^{\mathrm{c}}, H_{q}$, and $H_{q}^{(2)}$, for two different state points $\mathrm{S} 1$ and $\mathrm{S} 2$, as obtained from fits of $F_{\mathrm{BB}}(q, t)$ to Eq. (4). The state points are $S 1:\left(x_{\mathrm{B}}=0.3, T=0.4\right)$ and $S 2:\left(x_{\mathrm{B}}=0.3\right.$; $T=0.5)$. The characteristic times $\tau$ for $\mathrm{S} 1$ and $\mathrm{S} 2$ are respectively 1100 and 67 . The $f_{q}^{\mathrm{c}}$ for the homopolymer $\left(x_{\mathrm{B}}=1\right)$, as obtained from fitting to Eq. (3), is also shown for comparison in panel (a). The dashed line in panel (c) indicates zero value for $H_{q}^{(2)}$.

on the distance of the state point $\left\{\mathbf{x}^{n}\right\}$ to the transition point $\left\{\mathbf{x}_{c}^{n}\right\}$. As shown in Fig. 3 $b$, Eq. (44) provides an excellent description of the simulation data for $F_{\mathrm{BB}}(q, t)$ in the blend. It is worthy of remark that the observed convex-to-concave crossover is present in the higher-order MCT scenario 22, 27, 28]. It is indeed one of its main signatures and differentiates it from other theoretical frameworks 22]. The pure logarithmic decay observed at some $T$ - and $q$-values is also present in the higher-order MCT scenario, which predicts 22, 27, 28] lines in the control parameter space with $H_{q}^{(2)}=0$.

Figs. [5 and [6] show the values of $f_{q}^{\mathrm{c}}, H_{q}$, and $H_{q}^{(2)}$ obtained from the fits to Eq. (44) of $F_{\mathrm{BB}}(q, t)$ for two sets of state points $[\mathrm{S} 1, \mathrm{~S} 2]$ and $[\mathrm{S} 3, \mathrm{~S} 4]$. The coordinates of such state points are $\mathrm{S} 1:\left(x_{\mathrm{B}}=0.3, T=0.4\right)$, $\mathrm{S} 2:\left(x_{\mathrm{B}}=0.3, T=0.5\right), \mathrm{S} 3:\left(x_{\mathrm{B}}=0.6, T=0.4\right)$, and $\mathrm{S} 4:\left(x_{\mathrm{B}}=0.6, T=0.6\right)$. The fact that a common value of $f_{q}^{c}$ is found for the states of each set, together with the scaling behavior of $H_{q}$ (see below) would be consistent with the existence, for each set, of a nearby MCT higher-order transition $(\lambda=1)$ or a fold transition with $\lambda \lesssim 1$. Interestingly, $f_{q}^{c}$ shows no strong modulation but a nearly monotonous decay (see Figs. 5 and 6 a), resembling the qualitative behavior observed at $\overline{\mathrm{A}_{3}}$ - and $\mathrm{A}_{4}$-transitions in short-

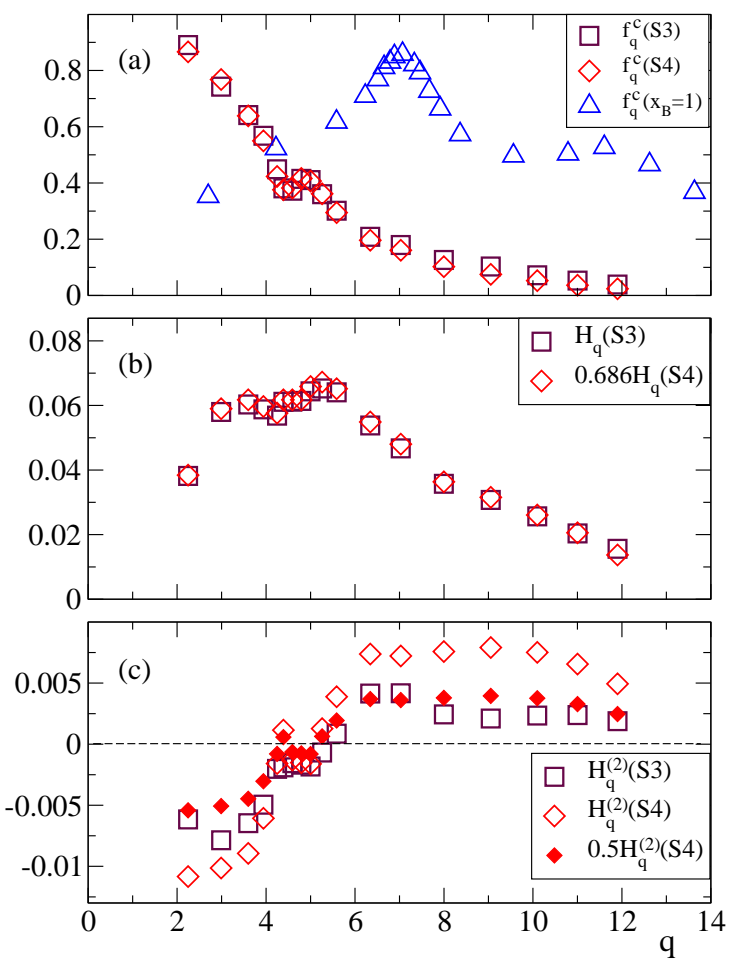

FIG. 6: As Fig. 5 for the state points $S 3:\left(x_{\mathrm{B}}=0.6\right.$, $T=0.4)$ and $S 4:\left(x_{\mathrm{B}}=0.6 ; T=0.6\right)$. The characteristic times $\tau$ for S3 and S4 are respectively 200 and 7.4.

ranged attractive colloids 13, 15, 19, 22, 23.

According to MCT 15, 22], the coefficient $H_{q}$ in Eq. (44) factorizes as $H_{q}=C\left(\left\{\mathbf{x}^{n}\right\}\right) \tilde{H}_{q}$, where $\tilde{H}_{q}$ only depends on $q$, and the $q$-independent term $C\left(\left\{\mathbf{x}^{n}\right\}\right)$ depends on the state point. Hence, the values of $H_{q}$ obtained for different state points close to the transition point must be proportional. This scaling behavior is indeed displayed by simulation data, as demonstrated in Figs. [5b and 6 $6 \mathrm{~b}$. As for $f_{q}^{\mathrm{c}}$, the qualitative $q$-dependence of $H_{q}$ resembles that observed at higher-order MCT transitions in short-ranged attractive colloids.

Also resembling MCT predictions for the higherorder scenario [15, 22], the coefficient $H_{q}^{(2)}$ is smaller than $H_{q}$, and does not obey scaling. Indeed any test of scaling behavior over a wide $q$-range for $H_{q}^{(2)}$ is unsatisfactory (see two examples in Figs. [5 and 6r). Apparently, the value of $q$ for which $H_{q}^{(2)}=0$ is dependent on the state point, in agreement with MCT expectations.

The striking dynamic analogies of the bead-spring polymer blend here investigated with the higher-order MCT scenario, which has been derived for shortranged attractive colloids, provide connections with different research fields. In these latter systems, in a narrow range of $\phi$ and $T$, anomalous relaxation originates from the competition between two arrest mechanisms of very different localization lengths: reversible bond formation induced by the short-ranged attractive potential, and hard-sphere repulsion 14, 15, 17, 31]. The dynamic analogies between so different systems suggest that this anomalous scenario might be 
a general feature of systems with several competing mechanisms for dynamic arrest, and should motivate theoretical approaches within the MCT framework. In the case here investigated, we suggest bulk-like caging and confinement as the two competing mechanisms for arrest of the fast B-component. While the former is induced by the neighboring B-particles, the latter is induced by the matrix formed by the chains of the slow A-component.

It is worthy of remark that, due to chain connectivity, any given particle has always neighboring particles of its same species. Hence, competition between bulklike caging and confinement for the dynamics of the fast component occurs even at high dilution of the Bparticles. Indeed, all the reported anomalous features are observed in a composition range from $x_{\mathrm{B}} \lesssim 0.8$ to, at least, the smallest investigated value $x_{\mathrm{B}}=0.1$.

Results reported here must not be understood as a proof of the existence of higher-order MCT transitions (or fold transitions with exponent parameter $\lambda \lesssim 1$ ) in the system here investigated. A proper answer to this question could only be provided by solving the corresponding MCT equations. Still, it is worth mentioning that recent theoretical calculations by Krakoviack 32. have reported a higher-order MCT transition for a binary mixture of mobile and static hard spheres, which can be seen as a simplified model for liquids in confining media with interconnected voids. This system, where competition between bulk-like dynamics and confinement occurs, is a priori closer to polymer blends than short-ranged attractive colloids. This result supports the hypothesis of an underlying higherorder MCT scenario in the model here investigated.

\section{CONCLUSIONS}

We have carried out simulations on a bead-spring model for polymer blends. This model displays nonconventional glassy dynamics. Mean squared displacements and density-density correlators for the fast component exhibit many of the precursor effects characterizing nearby MCT higher-order transitions, or fold transitions with exponent parameter very close to unity.

These anomalous features are analogous to those observed for short-ranged attractive colloids, for which a higher-order MCT scenario has been derived, suggesting that such features might be generally present in systems with several competing mechanisms for dynamic arrest. We suggest bulk-like caging and confinement for the case of polymer blends. Chain connectivity extends competition between both mechanisms over a wide range of blend compositions. The strong resemblance of dynamic correlators with those recently reported in fully atomistic simulations of a real polymer blend strongly supports the dynamic picture here presented. These results open the possibility of new theoretical approaches for investigating polymer blend dynamics.

\section{ACKNOWLEDGEMENTS}

We thank W. Götze, E. Zaccarelli, F. Sciortino, and T. Franosch for useful comments and discussions. Support from the projects NMP3-CT-2004-502235 (SoftComp), MAT2004-01017 (Spain), and 206.215$13568 / 2001$ (GV-UPV/EHU Spain) is acknowledged.

* Corresponding author: wabmosea@sq.ehu.es

[1] T. P. Lodge and T. C. B. McLeish, Macromolecules 33, 5278 (2000).

[2] R. Kant, S. K. Kumar, and R. H. Colby, Macromolecules 36, 10087 (2003).

[3] T. R. Lutz, Y. Y. He, M. D. Ediger, H. H. Cao, G. X. Lin, and A. A. Jones, Macromolecules 36, 1724 (2003).

[4] A. C. Genix, A. Arbe, F. Alvarez, J. Colmenero, L. Willner, and D. Richter, Phys. Rev. E 72, 031808 (2005).

[5] G. S. Grest and K. Kremer, Phys. Rev. A 33, R3628 (1986).

[6] C. Bennemann, W. Paul, K. Binder, and B. Dunweg, Phys. Rev. E 57, 843 (1998); C. Bennemann, J. Baschnagel, and W. Paul, Eur. Phys. J. B 10, 323 (1999).

[7] For comparison, the original Lennard-Jones binary mixture investigated by Kob and Andersen [W. Kob and H. C. Andersen, Phys. Rev. E 51, 4626 (1995)] has $\phi=0.59$ with the definition of packing fraction given above.

[8] D. Frenkel and B. Smit, Understanding Molecular Simulation (Academic Press, San Diego, 1996).

[9] W. Götze and L. Sjögren, Rep. Prog. Phys. 55, 241 (1992).

[10] W. Götze, J. Phys.: Condens. Matter 11, A1 (1999).

[11] M. Aichele and J. Baschnagel, Eur. Phys. J. E 5, 229 (2001); ibid. 5, 245 (2001).

[12] S.P. Das, Rev. Mod. Phys. 76, 785 (2004).

[13] E. Zaccarelli, G. Foffi, K. A. Dawson, S. V. Buldyrev, F. Sciortino, and P. Tartaglia, Phys. Rev. E 66, 041402 (2002).

[14] A. M. Puertas, M. Fuchs, and M. E. Cates, Phys. Rev. Lett. 88, 098301 (2002).

[15] F. Sciortino, P. Tartaglia, and E. Zaccarelli, Phys. Rev. Lett. 91, 268301 (2003).

[16] F. Mallamace, P. Gambadauro, N. Micali, P. Tartaglia, C. Liao, and S. H. Chen, Phys. Rev. Lett. 84, 5431 (2000).

[17] K. N. Pham, A. M. Puertas, J. Bergenholtz, S. U. Egelhaaf, A. Moussaid, P. N. Pusey, A. B. Schofield, M. E. Cates, M. Fuchs, and W. C. K. Poon, Science 296, 104 (2002).

[18] T. Eckert and E. Bartsch, Phys. Rev. Lett. 89, 125701 (2002).

[19] L. Fabbian, W. Götze, F. Sciortino, P. Tartaglia, and F. Thiery, Phys. Rev. E 59, R1347 (1999).

[20] J. Bergenholtz and M. Fuchs, Phys. Rev. E 59, 5706 (1999).

[21] K. Dawson, G. Foffi, M. Fuchs, W. Götze, F. Sciortino, M. Sperl, P. Tartaglia, T. Voigtmann, and E. Zaccarelli, Phys. Rev. E 63, 011401 (2000).

[22] M. Sperl, Phys. Rev. E 68, 031405 (2003).

[23] W. Götze and M. Sperl, J. Phys.: Condens. Matter 15, S869 (2003).

[24] A MCT transition of order $n, \mathrm{~A}_{n}$, is characterized by $n$ solutions of the critical non-ergodicity parameter, only the highest value being physical.

[25] S. H. Chong and M. Fuchs, Phys. Rev. Lett. 88, 
$185702(2002)$

[26] As also found for fold transitions [9, 10, 12], the coefficients $h_{q}$ and $h_{q}^{(2)}$ are in antiphase with $f_{q}^{\mathrm{c}}$.

[27] W. Götze and R. Haussmann, Z. Phys. B 72, 403 (1988).

[28] W. Götze and M. Sperl, Phys. Rev. E 66, 011405 (2002).

[29] Strictly speaking, sublinear behavior is derived from MCT by expanding the mean squared displacement just to first-order.

[30] A correction to $f_{q}^{\mathrm{c}}$, of the order of the distance to the transition point, is neglected in Eq. (4)

[31] A recent work [E. Zaccarelli et al., J. Chem. Phys. 124, 124908 (2006)] has reported anomalous relaxation features for a short-ranged attractive colloid with a constraint in the maximum number of bonds for each particle (note that the standard case is unconstrained). Hard-sphere repulsion and reversible gelation of a bonded network have been suggested as competing arrest mechanisms for this system.

[32] V. Krakoviack, Phys. Rev. Lett. 94, 065703 (2005). 